University of Nebraska - Lincoln

DigitalCommons@University of Nebraska - Lincoln

Faculty Publications, Department of Psychology

Psychology, Department of

1989

Creativity and Perception

John H. Flowers

University of Nebraska-Lincoln, jflowers1@unl.edu

Calvin P. Garbin

University of Nebraska-Lincoln, cgarbin1@unl.edu

Follow this and additional works at: https://digitalcommons.unl.edu/psychfacpub

Part of the Psychiatry and Psychology Commons

Flowers, John H. and Garbin, Calvin P., "Creativity and Perception" (1989). Faculty Publications, Department of Psychology. 453.

https://digitalcommons.unl.edu/psychfacpub/453

This Article is brought to you for free and open access by the Psychology, Department of at DigitalCommons@University of Nebraska - Lincoln. It has been accepted for inclusion in Faculty Publications, Department of Psychology by an authorized administrator of DigitalCommons@University of Nebraska - Lincoln. 
Published (as Chapter 8) in Handbook of Creativity, edited by John A. Glover, Royce R. Ronning, and Cecil R. Reynolds (New York \& London: Plenum Press, 1989), pp. 147-162. Copyright (c 1989 Plenum Press (Springer). Used by permission.

\title{
Creativity and Perception
}

\author{
John H. Flowers and Calvin P. Garbin \\ Department of Psychology, University of Nebraska-Lincoln, Lincoln, NE 68588.
}

Informal thought about the nature of mental operations important to creative human behavior suggests that perceptual processes are of considerable importance. The ability to "see relationships among elements" is an attribution commonly made toward authors of major scientific discoveries or of noteworthy artistic achievements. For example, Shepard (1978, 1981) documented self-reports from several creative scientists and authors that strongly emphasize the role of visual imagery and the manipulation of visual codes in the creative process.

Given the anecdotal and self-report evidence for a relationship between creative behavior and aspects of perceptual processing, it initially may seem surprising that there is a notable void in either research or theoretical articles specifically focused on these issues. In preparing this chapter, for example, we noted that, during the last six volumes of the Journal of Creative Behavior, there was only one title that included the word perception, and that paper (Goodman \& Marquart, 1978) was limited to a one-page abstract. In addition, we noted that among seven current textbooks in perception that presently reside on our bookshelves, none contain the term creativity in their indexes, nor is the term creative ability addressed at any point in the texts. Although references to the term perception occasionally can be found in indexes of monographs specifically dealing with the topic of creativity, most of these references refer to research related to specific theories about individual differences in perceptual styles or processing modes, as opposed to broader contemporary issues of perceptual processing. Clearly, most researchers in the field of perception have not touched upon the topic of creativity, and relatively few researchers in creativity have chosen to integrate their work with perceptual issues.

\section{Why Have Perceptual Psychologists Had Little to Say about Creativity?}

One reason that may have inhibited psychologists who were studying aspects of perception from becoming involved in research on creativity is the fact that the term itself is not viewed as a scientifically "tight"' concept of the variety preferred by relatively "operationalistic" behavioral scientists. As has been noted in earlier research (e.g., Stein, 1956; Taylor, $1960)$, as well as the contributors to this volume, providing an easily agreed upon operational definition of creativity that can be related to specific aspects of observable behavior or specific information-processing operations is problematic. Although there is general agreement among researchers in human thinking 
that a key component of creativity is the process of generating novel mental representations, assessment of creativity by society is generally done on the basis of product; that is, does a particular solution, invention, discovery, or artistic contribution meet the joint criteria of novelty and worthwhileness? Obviously, a wide variety of mental operations and processes could contribute to the characteristics of a product that elicit those subjective evaluations.

The lack of existing literature explicitly relating issues in perception to the study of human creativity may also be attributable, in part, to fundamental differences in the "level of approach" among behavioral scientists who study fundamental characteristics of mental and behavioral processes common across individuals, as opposed to those having primary interest in differences among individuals. Both historically and at present, the majority of research efforts directed at understanding perceptual processes has been directed at theories, models, and descriptions of behavior that apply to perception in general, as opposed to individuals. The very term creativity, on the other hand, denotes an attribute that individuals presumably possess (or at least exhibit) in different amounts, hence, its study generally assumes an individual differences approach.

In our view, however, neither the fuzziness attributed to the definition of creativity nor its degree of association with ideographic rather than nomothetic approaches is as responsible for the lack of an existing body of literature relating creativity and perception as is the fact that perception traditionally has been studied as a process of organizing information within the nervous system, whereas studies of mental processes associated with creative behavior usually imply the generation of novel representations of information within the nervous system. At first inspection, these two classes of mental activity seem to have little to do with each other-or worse yet, they may even be viewed as incompatible operations.

\section{Is Perceptual Organization Incompatible with Creative Thought?}

Although individual theories of perception may differ substantially in their emphasis upon the importance of stimulus structure versus mental organizational processes (see, e.g., Hochberg, 1981, for a review), most theories approach perception as an information reduction process whereby noisy, variable, and impoverished patterns of environmental energy become resolved into stable and consistent internal representations optimal for human performance. Terms such as perceptual organization and perceptual constancy reflect emphasis on the information reduction processes. The latter term, in particular, emphasizes the role of perception in providing an individual with similar or identical mental representations of events that may have widely varying physical representations in the environment.

If a major function of "efficient" perceptual processing is to provide perceptual constancy, as well as to encourage different observers to obtain similar or identical representations from common environmental stimuli, then it does indeed seem that this organizational aspect of perception works against the generation of novel representations. One might therefore predict that some measures of perceptual performance that tap perceptual organizational processes would be negatively related to measures of creative ability. A potential consequence of such a relationship would be a positive association between perceptual deficiencies or handicaps and creative ability. Case study instances supportive of a relationship between perceptual handicaps and creative behavior can, in fact, be found in discussions of artistic and musical achievement. For instance, some of the innovative stylistic changes in Beethoven's later works are commonly attributed to the increasing severity of his hearing impairment.

However, there are many reasons to believe that some aspects of perceptual performance should be positively correlated with creative ability - or at least with the output of creative products. Many products of creative activity are not simple spontaneous generations, but result from effortful production, interspersed with the evaluation of feedback. Thus, handicapping the senses at a peripheral level (e.g., blindness or deafness) would certainly disrupt the ability to evaluate visual and auditory productions as they are being created. Despite what has been said about Beethoven's deafness, it seems absurd to predict that there would be a general negative relationship between basic measures of auditory sensitivity and frequency discrimination ability and creative output of music among composers, or a negative relationship between visual acuity measures and creative output of painters. 


\section{The Importance of Executively Controlled Processing}

In our view, however, there are other aspects of perception for which performance measures ought to relate positively to creativity -in particular, processes under the conscious control of the perceiver. These include the ability to control various aspects of selective attention, to control figural organization when ambiguous sensory data are presented, to perform manipulations of internal visual and/or auditory representations of perceptual information, and the ability to equate perceptual experiences obtained from different sense modalities (cross-modal abilities). Although the earlier stages of perceptual processing leading to perceptual organization (e.g., sensory transduction, feature extraction, figural synthesis) typically are viewed as involuntary processes not under executive control, it is clear that perceptual organization and pattern recognition are influenced jointly by both involuntary or automatic and executively controlled processing.

At the level of perceptual organization, consider the examples of the Necker cube and of the reversible or ambiguous figures commonly found in most introductory psychology textbooks as well as in textbooks on perception (some examples are given in Figure 1). In each of these examples, there are contextual cues that cause a particular organization of the object to be perceived upon initial inspection. In most versions of these objects, observers have an ability to reverse the organization through voluntary changes in visual attention, although the time and effort required for such reversals, as well as which of the possible organizations is most likely to be initially seen, can be greatly influenced by modifying the contextual cues supporting either of the organizations. With some modifications, reversal becomes virtually impossible, and thus only one interpretation is obtained. Executively controlled processes can, within limits, significantly modify figural synthesis and organization, allowing a single physical stimulus to have multiple perceptual representations. Such processes may thus play a role in the generation of novel mental representations of information, which, in turn, could form the basis for creative products. Indeed, it is this kind of processing that appears to contribute to high-performance levels on tests, such as Guilford's (1967) divergent production test, that are specifically aimed at measuring individual differences related to creativity.
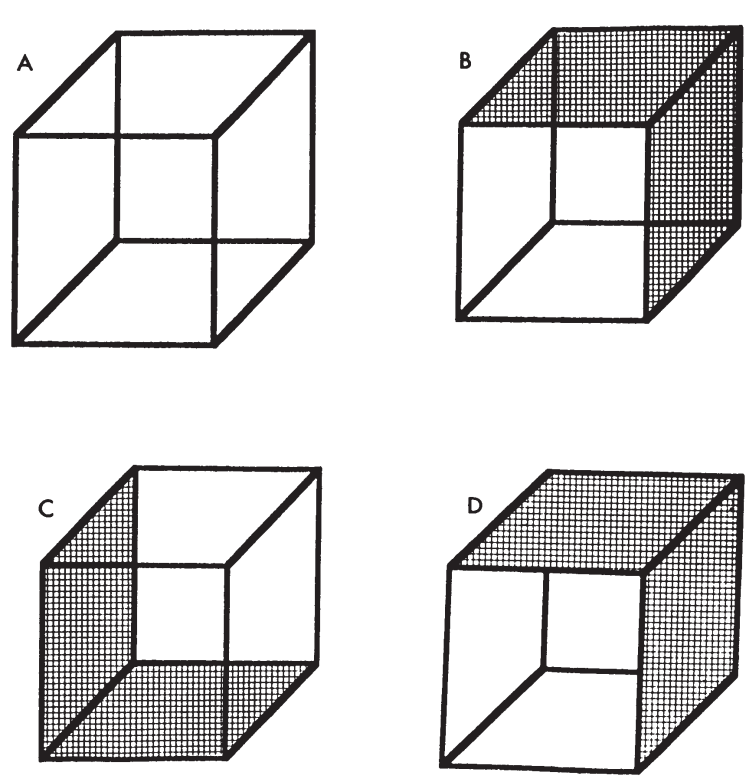

Figure 1. The Necker Cube-a familiar example of how controlled visual attention can change perceptual organization. In Example A, where the cube is essentially "transparent," two distinct organizations of depth can be obtained with moderate ease. In Example B, the shading and obscuring of the internal contours provide additional context making one organization dominant, although the second organization can still be obtained with effort. In Example C, shading of other parts of the figure makes the opposite depth organization dominant. In Example D, where the internal contours are totally removed, the alternative depth organization cannot be obtained at all (by typical subjects), even with considerable mental effort. Note also that, after viewing cube C, it is easier to view cube A (and even cube B) in the same organization as is dominant for cube $\mathrm{C}$.

Thus, we see two distinct categories of perceptual processes for which individual variation among different observers might well relate to creativity. The first category contains the largely involuntary processes of perceptual organization. These are information-reducing processes that promote stability and organization of percepts, and thus normally tend to act against the formation of novel representations of information. The second category of perceptual processes, those under executive control of the observer, serve to modify and control the actions of the involuntary processes and also may serve to encourage the generation of novel representations of information. Extensive data exist on how each of these classes of processes affects performance as a function of stimulus and task parameters. Unfortunately, the data base on individual differences among either of these categories of processes is limited. Thus, it is difficult to re- 
late directly specific empirical findings from existing perceptual studies to either the prediction of creative behavior in individuals or to modification of the environment to encourage creativity. We feel this indicates a need for additional basic research in individual differences per se, as well as research that directly relates these individual differences to creative ability.

\section{A Configural Conception of Creativity}

The particular topics (and perceptual tasks) that we will be discussing are selected largely because they focus on the joint role of involuntarily and executively controlled processes in perception. We are guided by a broad conception of creativity that assumes that there are essentially three factors that influence an individual's creative potential. One involves the relative "looseness" of involuntary organizational processes. An individual for whom the involuntary processes operate somewhat less deterministically (but perhaps less efficiently) is more likely to represent environmental data mentally (as well as data recalled from memory) in novel ways. The potential relationship between schizophrenia and creativity (Keefe \& Magaro, 1980; Prentky, 1979) would seem to illustrate this factor.

The second factor involves the power of executively controlled processes, such as spatial selective attention, manipulation of mental images, and controlled cross-modal representation. Presumably, an individual having superior executive control of these processes is able to produce novel representations of information through effortful construction and modification of mental representations. This concept of "creativity through controlled mental effort" is very different from the concept of creativity attributable to "loose organizational processes." For example, schizophrenics and individuals with schizotypic patterns of cognitive activity are notably weak on performance measures that presumably tap executively controlled mental processes.

The third factor, we feel, is of importance, particularly to creative thought that involves sudden insight, that involves processes not under executive control nor driven by sensory data, but that produces seemingly spontaneous mental representations, often involving visual imagery. Based largely upon self-reports of creative scientists and authors, Shepard $(1978,1981)$ attributed this aspect of creative activity to the same perceptual mechanisms that are normally coupled to the analysis of sensory input, and that are responsible for many aspects of perceptual organization. According to Shepard, these highly evolved and specialized perceptual mechanisms have the ability to operate upon data other than that obtained from normal sensory input, when decoupled from the sensory environment, as in such altered consciousness states as dreams. It is conceivable that the ability to use such spontaneously generated mental constructions in creative thought could be relatively independent of an individual's power of executively controlled mental operations, but positively correlated with the strength of perceptual organizational processes.

This three-factor view of creativity, directly based upon perceptual mechanisms, suggests therefore, a highly configural, nonlinear relationship between creative ability in the population and specific individual differences in mental processes. By looking - from an individual differences perspective-at various perceptual tasks that seem likely to tap differentially involuntary organizational processes and executively controlled processes, researchers may be in a better position to form a more detailed model relating specific representational and transformational processes to creative behavior. Additionally, such research might guide development of programs or products for the enhancement of creative ability.

\section{Impairment of Sensory Processes}

One of the most highly noticeable individual difference variables in perception is that of impairment of one or more of the primary senses - at least insofar as distinguishing normal individuals (including prosthetically correctable persons) from those who have apparent handicaps. Case study analyses of creative output from visually or auditorily handicapped individuals is a topic that deserves intensive study on its own; our limited mention of the topic here is merely to recognize that the broad categories of differences in perceptual and cognitive processes that characterize adaptation to severe defects in either hearing or vision obviously can affect the creative process, albeit in complex ways. It is a well- known principle of developmental psychobiology that prolonged deprivation of sensory experience, from birth or early in life, can result in permanent changes in neural structures that often prevent full recovery of function, even if 
the handicap is later repaired. Furthermore, neural structures may adapt functions qualitatively different from those assumed by corresponding structures in sensorily intact individuals. In human behavior, the consequences of sensory handicaps may affect aspects of memory and cognition that depend upon the use and manipulation of auditory or visual "codes," in addition to the ability to gain information from the environment. Recognition of this fact has led researchers and educators to develop specialized educational programs for hearing-impaired individuals that are specifically adapted to differences in modes of information processing among such individuals. In addition to producing performance differences per $s e$, differences in memory codes, attention, and other control processes attributable to early sensory handicaps obviously have the potential for producing products that are novel when compared with norms of the general population of nonhandicapped individuals.

We see two major problems, however, in extrapolating general conclusions about creativity from the study of the perceptually handicapped. The first problem is the extreme degree of heterogeneity in types and degree of impairment among such individuals; not to mention potential differences in compensatory processes among individuals who share relatively similar physical impairments and developmental histories. The second problem is that the ease of retrieving examples of perceptually handicapped creative individuals from memory makes the topic ripe for attributions of correlations between the handicap and the creativity that may not be warranted (e.g., Kahneman \& Tversky, 1973). One may be able to learn a great deal about the creative processes of a given handicapped individual through careful case study, protocol analyses, and other techniques, and such research potentially could be of tremendous value to the development of special therapeutic and educational approaches. As a simple individual difference variable to be applied for understanding the characteristics of creativity in the general population, the handicapped-nonhandicapped dichotomy does not, on the other hand, appear to be very useful.

\section{Environmental Constraints on Sensory Input}

It may be more useful to consider the effects, within unimpaired individuals, of different levels of sensory stimulation upon creative thought processes. In particular, the fact that common resources are used in executive control of mental representations and in the processing of corresponding forms of sensory data implies that perception within a particular modality may interfere with thought processes sharing a common form of representation. For instance, the generation and the manipulation of visual images are inhibited when a task requires processing visual input, and manipulations of linguistic representations may be inhibited by tasks requiring the processing of speech. The interference between perceptually generated and internally generated codes that share a common modality easily can be demonstrated by using a task developed by Brooks (1968). In this task, subjects are required to imagine a moving dot traveling around a mental image of a block drawn letter, such as a capital F (see Figure 2), and to respond orally yes or no based upon whether each comer constitutes an external angle (requiring a "right turn" on the part of the dot) or an internal angle (requiring a "left turn"). Time to complete the circuit with the oral response mode is generally much less than that required for the same task when subjects must check off Ys versus Ns in a visually presented answer sheet. However, when subjects are presented with a linguistic search task, requiring them to indicate whether successive words in memorized sentences are nouns versus other parts of speech, the

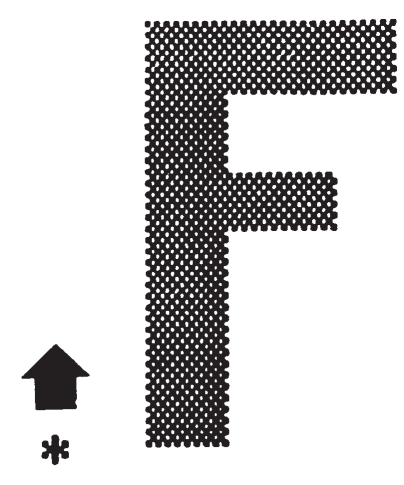

Figure 2. An illustration of the sort of "imaginary stimuli" used by Brooks (1968) to study the effect of verbal or visually guided response processes on the ability to search visually coded information in active memory. Subjects were required to mentally construct an image of a letter, such as this capital F, and to indicate whether each comer passed by the imaginary asterisk consisted of an external or an internal angle. Subjects could perform better on this task if an oral yes-no response was used than if a visually guided manual response system was employed, indicating that manipulation of visual codes shares resources with visual perception. 
visually guided check-response mode produces considerably faster searches than does the oral yes-no mode. Modality specific interference between imagery and perceptual detection tasks also has been demonstrated by Segall and Fusella (1970), who asked subjects to construct mental images, such as the appearance of a tree (visual) or the ringing of a telephone (auditory), and showed selective deficits in the ability to detect weak auditory or visual signals.

Because documented self-reports of mental events associated with creative thought often include extensive use of mental imagery (Shepard, 1978, 1981), it seems plausible that creating an environment that minimizes potentially interfering sensory input might be useful in facilitating manipulations of mental image processing and, hence, contribute to creative thought. Additional research in how regulation of stimuli (visual and linguistic feedback from a display screen on a word processor) can affect the speed and quality of creative output is one area of applied perceptual research that has current value for product development. An increasing number of products are being released into the personal and professional software markets that are designed to aid the initial stages of manuscript planning and organization. These so-called idea processors are aimed specifically at the facilitation of creative output for a variety of applications (Kellogg, 1986). With the increasing use of small computers in a variety of settings, one might expect the developments of similar products to continue. One attribute of at least some of the existing hardware is that users are prevented from viewing previously entered text $-\mathrm{a}$ design feature that seems to assume that the availability of visual feedback, or at least the existence of printed text that stimulates the visual system of the user, is detrimental to performance.

As Kellogg (1986) pointed out, evaluative research on these products and on prototypes for future related software aids is woefully lacking. The same can be said for research that is not tied to a specific product but that provides general information about the production as a function of the presence of perceptual stimuli of, for example, text, figural design, and metaphorical or analogical relationships. Based upon the implications of such studies as those of Brooks (1968), it may be the case that the presence of auditory or visual stimuli, while an individual is attempting to "be creative," can have certain interfering stimulus-related costs for mental representation and transformation, and, hence, inhibit creative per- formance. The knowledge base that is generated from such a research effort might prove very useful in the development of both text-related products and picture-related software aids used in computer-assisted design. More generally, however, such knowledge might prove useful in the development of techniques for fostering increased output of creative activity in a much wider range of settings, through the teaching of specific strategies for minimizing perceptual interference with image-based mental operations and other forms of mental code transformations.

\section{Altered and Transitional States - Decoupling Perceptual Mechanisms from Sensory Input}

Our discussion above has concerned the overlap in resources between mechanisms normally tied to the involuntary processes of analyzing and organizing sensory stimulation and those of executively controlled construction and manipulation of visual and auditory images. A related issue concerns the potential role of the perceptual mechanisms normally driven by sensory input during unaltered states of observer consciousness, when those mechanisms are decoupled from sensory input. Dream states, and perhaps some drug-induced states, represent examples of such a decoupling. Shepard $(1978,1981)$ argued that transitions from such states represent a fertile ground for the development of creative ideas, because the perceptual mechanisms automatically linked to organizing the sensory world (which are normally transparent to our conscious experience) run "on their own," occasionally constructing novel and useful percepts and images from fragments of internal neural noise and loosely guided consultations with memory. According to Shepard (1981), contact with the linguistic system allows the abstract images and relationships to be translated into communicable form. Shepard clearly viewed the mechanisms of perceptual organization that involve spatial relationships in particular as a powerful source of general knowledge about relationships that can be analogically applied to invention and problem solving:

The creative productions of a brain presumably stem from whatever intuitive wisdom, whatever deep organizing principles have been built into that brain as a result of the immense evolutionary journey that has issued in the formation of that brain. If the arguments sketched out in this chapter have any merit, the most basic and powerful innate intuitions and principles un- 
derlying verbal and nonverbal thought, alike, may well be those governing the relations, projections, symmetries, and transformations of objects in space. (Shepard, 1981, p. 339)

Thus, Shepard described a very direct relationship between perceptual processes and creative thought, making the claim that (1) implicit knowledge of visual relationships among objects, and rules for transforming those relationships, may constitute the fundamental mental operations inherent in much of creative thought, and (2) that decoupling certain normally involuntary processes from their data source may allow those same mechanisms to operate as a primary generator of creative thought. It seems apparent from Shepard's descriptions of self-report data that this form of creative thought is not of the controlled variety (as discussed in the previous section) but is the product of involuntary mental operations that lead to spontaneous insight. Thus, the effects of decoupling of normal sensory input during alternative states of consciousness should be viewed as distinct from restricting sensory input in a normal-waking-state individual in order to prevent interference with controlled manipulation of perceptual codes.

In popular religious and scientific circles, vast amounts have been written about alternative states of consciousness that involve such decoupling of sensory input, although, in our view, it is sometimes difficult to determine into which of those circles a given piece of research/literature should be categorized. It is also apparent that, throughout much of the history of civilization, some human beings knowingly have exploited alternative states (including dreams) as a deliberate strategy for fostering creative behavior - not to mention the use of alternative consciousness states as a causal construct for particular creative acts. Like research relating either handicaps or psychopathology to creativity, selection biases and the availability heuristic can play havoc with attempting to relate existing literature on alternate states and creativity either to individual differences in creative potential or to research into conditions that foster creativity.

It may, however, be profitable to study the extent to which individual differences in dream recall might relate to instances of insightful creative thought, as well as to individual differences in attention and perceptual organization. Such data could provide for a better empirical grasp on the degree to which information processing in dream or transitional states actually constitutes a major source of creative pro- ductions. Mental constructions occurring during an altered or transitional state can be useful only insofar as they are remembered, and insofar as they can be evaluated for application and worthwhileness. It may be that the degree to which decoupled automatic perceptual processes contribute to creative output has far more to do with facility in higher level cognitive processes, such as memory storage, retrieval, search, and comparison, than in individual differences in perceptual organization processes per se.

Consideration of the role of stimulus-decoupled perceptual organization processes as a fundamental source of creative thought, as Shepard proposed, leads to an interesting conjecture about the link between efficiency of perceptual organization and creativity-particularly with respect to how general cognitive defects, such as those associated with schizophrenia, might relate to creative thought. One common view of why individuals who possess some of the traits of schizophrenic thought might be viewed as creative is that deficiencies in the normal involuntary perceptual organization processes lead to an increased likelihood of an atypical representation of a perceptual event (see, e.g., Keefe \& Magaro, 1980). In other words, it is the anomalous organization of sensory input, coupled with sufficiently good higher order processes to evaluate the potential worthwhileness of a mental construction (or, alternatively, the opportunity for a peer to notice the worthwhileness of a product even if the cognitively impaired individual cannot perform the evaluation), that lead to creative output. However, creativity that is attributable to looseness in perceptual organization in the presence of stimuli is very different from creativity that is attributable to perceptual organization processes decoupled from normal sensory inputs. An increased frequency of transitions from hypnopompic or hallucinatory states (as might be reasonably expected to occur in association with certain psychotic disorders), combined with unimpaired (or even superior) mechanisms of perceptual organization, thus represents a potential alternative route for contributing to creative thought by some individuals who possess dispositions toward cognitive disorders. Moreover, the relative weakness or looseness in organizational processes and the ability to exploit involuntary organizational processes decoupled from sensory input are suggested as distinct individual difference variables, both of which might relate to creativity in the general population. 
Assessing Individual Variation in Perceptual Organization Ability

Our discussion thus far suggests that obtaining a better understanding of patterns of individual variation in perceptual organization tendencies might be highly useful in examining the role of perceptual organization processes in creative thought. Unfortunately, with the exception of various measures of embedded figures performance, Gestalt grouping processes and other related "automatic" processes of perceptual organization have not been subjected to systematic individual difference measurements. Performance measures on tasks, such as those involving embedded figures, often do not provide a convenient way of separating performance attributable to loose perceptual organization (failure to group), from performance attributable to strong, executively controlled focused attention (ability to break apart). It appears to us, however, that one should be able to separate the contributions of automatic organization and efficient focused attention, by selecting a combination of structurally similar tasks, including some in which perceptual organization is helpful to performance, and others for which perceptual organization is harmful.

As an example of such an approach, consider the example of the stimulus displays shown in Figure 3, used by Pomerantz, Sager, and Stover (1977). The

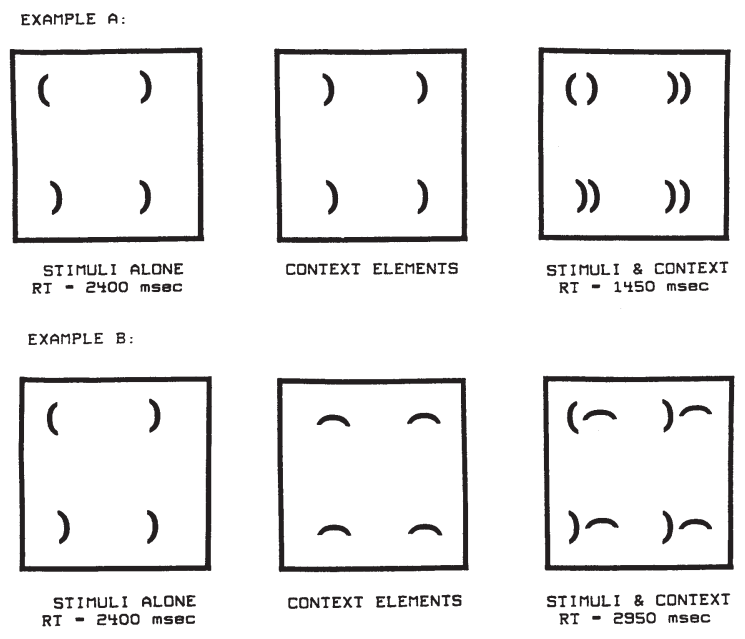

Figure 3. Examples of stimuli used in an "oddball quadrant" detection task employed by Pomerantz, Sager, and Stover (1977). In Example A, the addition of the context elements causes Gestalt grouping processes that make the unique quadrant more distinctive, whereas in Example B, the rotated context elements obscure the distinction (see text for details). task required of the subject was to detect the "oddball" quadrant as rapidly as possible, and choice reaction time (RT) was the dependent variable. In Example A, the baseline task involves choosing the oddly oriented single parenthesis, located in this example in the upper left. This display requires a quite effortful scrutiny as the mean RT obtained by Pomerantz et al. was $2,400 \mathrm{msec}$. However, adding the four extra context elements in the display leads to a perceptual grouping phenomenon that makes the oddball element stand out, reducing the RT by nearly a second (for a group average obtained with college student subjects). Now, consider the display shown in Example B. Here, different context elements are added (rotated parentheses) for which the result of organizational processes produces objects perceptually less distinct than the single parentheses, resulting in an average increase in RT of $550 \mathrm{msec}$. Thus, in these examples, we have two highly similar tasks, one for which Gestalt organization helps, one for which it hurts.

Presumably, individuals with weak organizational processes would fail to gain as much benefit from the helpful context, in comparison with more typical subjects, while performing tasks such as that of Example A. On the other hand, such subjects also might be less susceptible to interference from context stimuli that disrupt performance of typical subjects. In contrast, subjects possessing nominal automatic grouping processes, but exceptionally powerful executively controlled focal attentional processes, might exhibit far less interference from the harmful context, while still maintaining benefits from the helpful context.

A related set of classification or sorting tasks that have the potential for separately assessing looseness of involuntary perceptual organization and the strength of voluntary visual attention is the one used by Pomerantz and Schwaitzberg (1975). In this experiment, there were three different basic sorting tasks required of a subject, and the stimulus response mappings for each of these is shown in Figure 4. In Condition A (the control condition), subjects sorted stimuli on the basis of orientation of the leftmost parenthesis; the orientation of the right parenthesis was held constant. In Condition $B$, which required subjects to divide attention and attempt to ignore the irrelevant rightmost parenthesis, the response assignment was determined by the left parenthesis, and the right parenthesis varied in a manner orthogonal to the left parenthesis. In this condition, classification times were considerably slower 
CONDITION A (control)

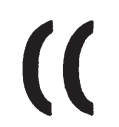

left response

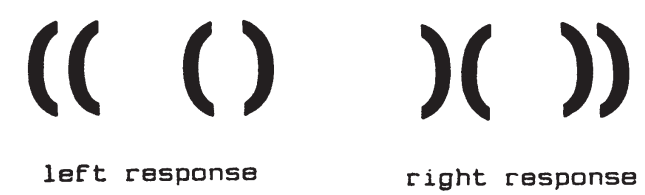

CONDIIION C (divided attention)

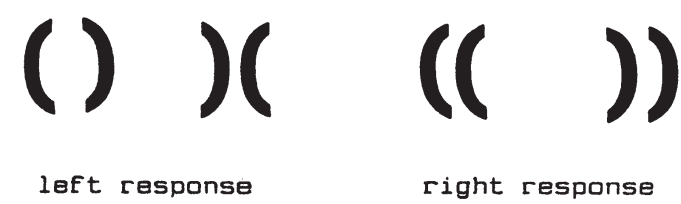

Figure 4. Examples of stimuli used by Pomerantz and Schwaitzberg (1975) in a task illustrating how Gestalt grouping affects selective and divided attention in a visual classification task (see text for details).

than those of the control condition, provided the spatial separation between the parenthesis pairs was close. As the spatial separation between the elements was increased beyond a single typespace in other stimulus sequences, the interference from the irrelevant right parenthesis diminished.

Condition $C$ required that subjects split the four possible pairings of parentheses into two groups, such that the response assignment was determined by the combination of both parentheses. Unlike either Condition A or Condition B, this task required an evaluation of both the leftmost and the rightmost parentheses. At close separations, such grouping seemed to occur involuntarily, as subjects reported perceiving the parenthesis pairs as single objects. In this case, classification times were actually shorter than for Condition B, because the objects assigned to each category appear to share common perceptual attributes (e.g., "the fat ones vs. the skinny ones"). However, at wider separations, in which involuntary grouping processes break down, subjects had to apply effortful divided attention processes, either to try to perceptually group the parenthesis pairs or to separately evaluate each parenthesis and apply a classification rule. A typical pattern of results for these three tasks is shown in Figure 5, in which sorting time is plotted as a function of separation of the parentheses. Again, these data are based upon the means of subjects' performances that were obtained by Pomerantz and Schwaitzberg (1975).

One might expect, however, that individual differences in the potency of involuntary organizational processes and individual differences in the executively controlled ability to break up perceptual configurations (as well as divide attention, in this case) would produce systematic differences in the functions from those shown in Figure 5. An appropriate application of psychometric scaling procedures to differences among such patterns, in addition to similar analyses of patterns in such tasks as the "oddball detection" examples in Figure 3, could lead to separate scales for strength of perceptual organization tendencies and power of voluntary selective attention.

To our knowledge, there is no existing research on patterns of individual differences in the costs and benefits of the configural effects that might be obtained by systematic administration of these or similar tasks to large numbers of individuals, in order to obtain an index of relative organizational power among different individuals that might be of use for determining its relationship to creative behavior. However, given that individual differences in more traditional measures of embedded figures tasks exist (even though the voluntary selective component has not been factored out), and given the recent evidence of the very unique and actually superior performance of schizophrenics (compared with normals) on tasks for which avoidance of grouping contributes to performance (Place \& Gilmore, 1980; Wells \& Leventhal, 1984), we believe that such individual differences exist, are potentially measurable, and probably do bear a relationship with other important cognitive attributes including creativity. Given that the presumed organizational looseness of schizophrenics is often related to creative thought in individuals who perhaps have a lesser degree of the deficiency (e.g., Keefe \& Magaro, 1980), the data on the psychiatric populations are of considerable interest and are encouraging for clarifying the "creativity-madness" association that has been around for centuries. 


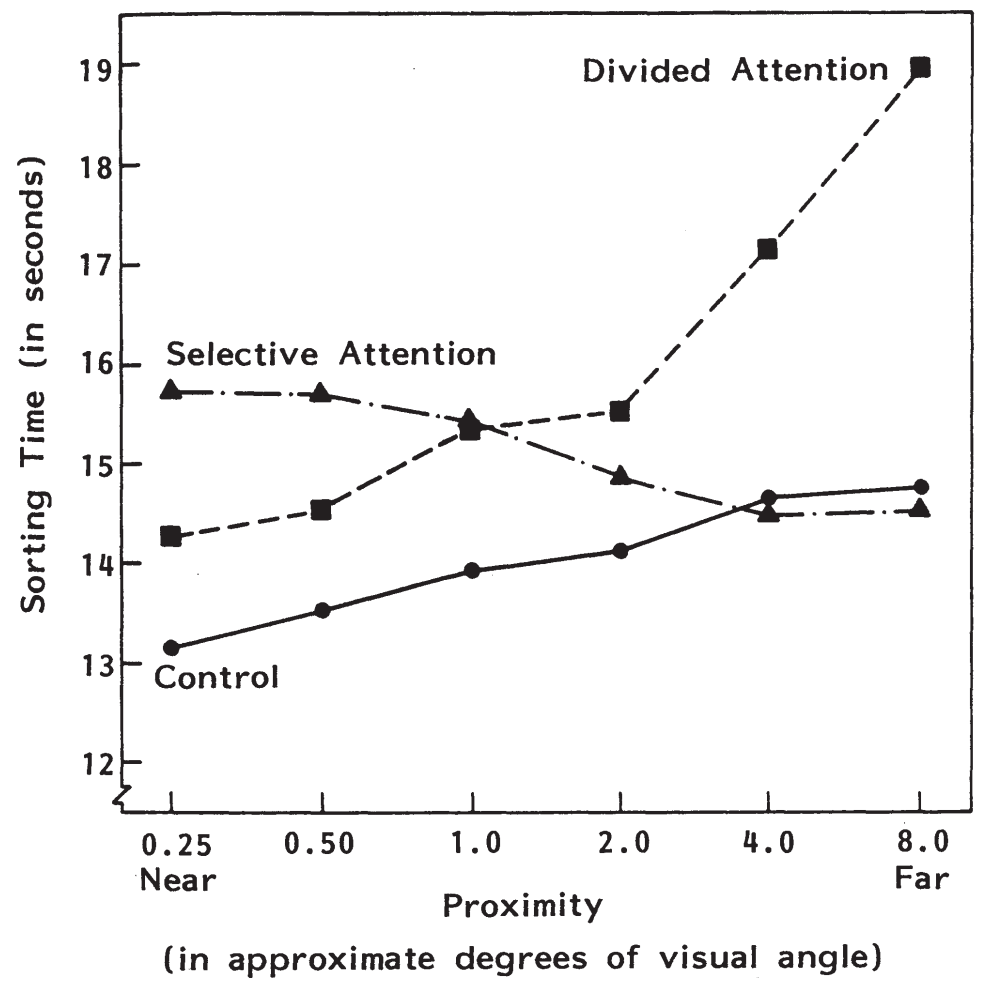

Figure 5. A graphical representation of results obtained by Pomerantz and Schwaitzberg (1975) for Tasks A, B, and C of Figure 4. Sorting times for decks of 32 cards displaying these stimuli are plotted as a function of the physical separation between the pairs of parentheses. Stimulus sets involving wide spatial separations between the two stimuli greatly slowed sorting in the divided attention task (Task C of Figure 4), while allowing subjects to avoid interference from perceptual grouping in Task B. Presumably the form of such functions might differ for subjects differing in the potency of their involuntary perceptual organization processes as well as the power of their controlled visual attention. Reprinted by permission of LEA Associates.

\section{Individual Variation in Intramodal Visual Code Transformation}

In contrast to our lack of knowledge about individual variation in perceptual organizing ability, psychometric tests that directly tap the ability of individuals to compare transformed visual objects (e.g., rotated block drawings, folded and unfolded block figures) have been available for decades (e.g., see Guilford, 1967). Individual variation in abilities to perform such operations is clearly measurable, and it is apparent that performance on such psychometric tests correlates with performance of mental tasks or problems that appear to be mediated by use of visual ana$\log$ codes.

One specific example is a study by Paivio (1978), who measured subjects' reaction times for determining which of two clock times (given as digital expressions) would produce the smallest angle between the hands of a standard analog clock. Paivio found that, in general, reaction time was a monotonic function of differences in the analog hand angles, providing strong evidence that the task was mediated by image comparison. In addition, however, subjects were divided into "high-imagery" and " 'low-imagery'? groups, on the basis of a median split of performance on a composite of Guilford's Block Visualization Test, the Minnesota Paper Form Board, and the Thurstone Space Relations Test. High-imagery subjects produced RTs that averaged about 1 second faster at each level of angular difference than low-imagery subjects. In contrast, a similar split of the subject population on the basis of a verbal fluency measure (Paivio, 1971) produced no differences between groups. This study as well as numerous others (e.g., see Paivio, 1971, 1978 for a review) provide relatively convincing evidence that individual differences in ability to perform top-down manipulation and transformation of visuospatial codes can be predicted effectively with existing psychometric instruments. 
Given the apparent importance of fluent visual coding in anecdotal reports of creative cognition, it seems intuitive that psychometric measures of such visual abilities should relate positively to measures of creative behavior, particularly for those individuals whose creative work is arrived at primarily through the effortful, voluntary route. One must keep in mind, however, that simple possession of a mental ability does not necessarily imply that ability will be used to produce novel and worthwhile products. Creative behavior requires an evaluative component for recognizing when a particular novel representation is of value. Given these considerations, and our configural view of the creative process, one should not expect extremely high linear correlations between measures of visual coding fluency and creative behavior per se.

One issue of importance in determining how specific information-processing characteristics relate to creativity is whether it is appropriate to view individual differences in performance of specific classes of perceptual tasks as measures of relatively narrowly defined information-processing abilities, or whether correlations among specific task performances suggest the existence of a general ability "factor." Guilford $(1981,1983)$ argued that processes, such as visual code manipulation and cross-modal transformations (which we will discuss presently), are all part of a general "transformational ability" that is a key component of creative thought. Indeed, one can make a rather strong psychometric argument for that position, as Guilford has done. On the other hand, perceptual psychologists who are aware that different neural structures may be involved in intramodal versus cross-modal transformations, and also that common neural structures may be involved in both voluntary and involuntary processing of visually coded information, are more likely to have interest in specific comparisons among tasks. One research question that bears upon the relationship between specific visual information-processing abilities and our configural model of creativity is whether fluency in controlled visual transformations might be related to the frequency of spontaneous insight in creative thought. We have thus far implied, as has Shepard $(1978,1981)$, that spontaneous novel constructions are basically the result of involuntary representational processes, decoupled from their normal sensory source of data. However, it must be noted that most of the anecdotal reports of such spontaneous insight, summarized by Shepard and others, have come from individuals who were likely to score very high on measures of mental abilities and who were (or still are) known to have put in large amounts of controlled mental effort on problems related to their creative achievements. The selection, refinement, and use of images spontaneously generated during transitional or altered states likely may depend upon effortful executive processes, suggesting that spontaneous insight should not be viewed as a totally involuntary occurrence. Moreover, mental activity that substitutes for the sensory signals that normally drive mechanisms of perception, insofar as they are influenced by memory activities, may be highly structured by previous effortful mental code manipulations. In short, spontaneous insight may not be so spontaneous and is conceivably quite closely related to mental transformation abilities of both intramodal and intermodal varieties.

\section{An Example of the Interplay of Involuntary and Executively Driven Creative Processes: Synesthesia and Cross-Modal Representations}

Previously, we have posited that these two very different processes might produce similar creative products. In this section, we will look at two related processes, one perceptual (automatic) and one cognitively mediated (executively controlled), that do indeed seem to lead to similar and potentially creative representations of the environment.

Theorists and artists long have recognized the correspondences, interrelationships, and interdependencies of the senses as they are used to capture information about the world. Aristotle and other early thinkers posited various relationships of cross-modal process or product, and Bishop Berkeley added his notion of their ontogeny. More recently, Stevens (e.g., 1959), Gibson (1966), and Marks (e.g., 1978, 1982) have provided more refined theories and hypotheses about the nature, workings, and meanings of these correspondences. Particularly for Marks, the evidence of artistic awareness and of creative use of these correspondences adds weight to various laboratory studies that are in support of the theoretical ideas of crossmodal equivalences and sensory unity. 
Most people are familiar with the use of onomatopoetic words, such as "hiss," "crack," and "woff," to convey the auditory characteristics of a sound as well as its semantic meaning. But the sounds of words also seem to convey other information. Kohler (1947) showed that most people matched the pseudowords "maluma" and "takete" with the rounder and the more angular of two line figures, respectively. As a demonstration, Marks (1978) replicated the finding using a production task in which two children were asked to draw the visual representations of these words. The drawing of takete was clearly the more angular for each person. As another example of how word sounds can supply nonauditory information, Sapir (1929) and later Newman (1933) asked subjects about the size of objects referred to by nonsense syllables and found the words containing the letter /a/ were judged to refer to larger objects than did those containing /i/. Finally, most people in our society are familiar with the relationships between colors and temperatures - red is "hot" whereas blue is "cool."

These types of correspondences are quite different from the wholly involuntary phenomena of true synesthesia, in which "a small minority of people experience a curious sensory blending, where stimulation of a single sense arouses a melange of sensory images" (Marks, 1978, p. 83). In addition to truly synesthetic individuals, there are widespread reports of involuntary synesthesia produced by various consciousnessaltering drugs, such as LSD and hashish.

As interesting and well-documented as true involuntary synesthesia is, it is not, in itself, a creative product, just as the novel representations of schizophrenic thought are not. Creative products require the additional processes of appropriate selection and presentation of those novel representations. Unlike the schizophrenics' art, which is often identified as creative after production, the synesthetics must choose whether or not to capture their mental representations and present their novel version of the world.

Furthermore, although loose perceptual organization is potential source of novel representations for synesthetics (as for schizophrenics), documentation of creative products from true synesthetics is notably lacking. In part, the lack of documented examples simply may reflect the relative infrequency of true synesthetic individuals in the population, relative to schizotypic or schizophrenic individuals. But from each of these sources of anomalous percepts, numerous prod- ucts have arisen. According to Marks (1978), synesthesia has enjoyed two periods of extensive study, the first 40 years of the twentieth century, and an earlier period during the nineteenth century. It was during this earlier period, when synesthesia was of interest not only to scientists and physicians but also to musicians, that creative products were introduced that clearly sprang from the perceptual phenomena. Perhaps the best known of these were the multimodal concerts that mixed music with colored lights and occasionally with odor. Louis-Bertrand Castel built the first light organ in 1735. This organ and others like it produced a particular colored light along with each note as the keys were depressed. Numerous pieces were written for and performed with these instruments. Laser light shows are a more recent version of this same artistic use of the close relationship between color and tonality to produce desired affective responses.

Also, during the nineteenth century and later, there were numerous linguistic expressions of synesthesia-like experiences, or synesthetic metaphors. The following examples of such poetry are taken from Marks (1978): Charles Baudelaire's “Correspondences," "perfumes fresh as children's flesh, sweet as oboes, green as prairies," and Arthur Rimbaud's "Sonnet of the Vowels" "A black, E white, I red, U green, $\mathrm{O}$ blue." Examples of synesthetic metaphor in literature are (from Marks, 1982): "the sound of coming darkness" (Poe); “a soft yet glowing light, liked lulled music" (Shelley); and "music suddenly opened like a luminous book" (Conrad Aiken).

On interesting point concerning these uses of color-sound correspondence and poetic metaphor (which is an important difference between these productions and those of schizophrenics) is that none of these authors appear to have been truly synesthetic themselves (although one may conjecture about those authors who had a history of narcotic use, e.g., Poe). Yet they were able to produce creative results using these correspondences in ways so compelling that we not only understand and agree with their meanings, but often do not even immediately notice that there are "crossed" or "mixed" modality-specific adjectives and nuances. Marks provided experimental evidence that most persons have (or can develop) a strongly internalized correspondence of cross-modal relationship between certain visual and auditory characteristics (primarily brightness and loudness). Thus, we see that an automatic perceptual process - the capabil- 
ity and tendency toward cross-modal associations is necessary for the appreciation of creative products generated by an executively controlled process.

In this discussion, we have tried to give an example of how knowledge of an infrequent, perceptual, and automatic phenomenon (synesthesia) can provide the impetus for the use of executively controlled processes to create artistic products. Finally, the appreciation of these creative products greatly depends upon the ability to "perceptually resonate" with those products, an ability that seems to be nearly universal and automatic.

\section{Summary and Conclusions}

We have argued that the generation of creative behavior can result from a combination of involuntary and executively controlled processes. These processes rely heavily on neural mechanisms and systems that have evolved primarily as perceptual systems. However, these systems, which embody highly sophisticated computational and inferential mechanisms, also serve to operate on mental codes actively retrieved from memory (e.g., conscious generation and modification of imagery). In addition, there is anecdotal evidence that these same mechanisms can be the source of spontaneously generated images or representations, when their normal driving source of sensory stimulation is decoupled, as in transitions from dreams or altered states. Such spontaneous generation, if appropriately selected and recognized as useful, perhaps can produce the sudden creative insights characterized by many self-reports from creative individuals.

According to our analysis, creativity thus can result from some combination of (1) novel percepts attributable to departures from the normal deterministic processes of perceptual organization, (2) effortful conscious mental activity involving manipulation and transformation of codes that generate novel representations, and (3) spontaneous generation of novel representations. Because the relative contributions of each of these to a specific creative achievement presumably varies markedly across both situations and individuals, the relationship between specific cognitive abilities or characteristics of processing and the likelihood of an individual's producing products judged to be creative is highly configural and thus difficult to measure. The configurality of factors is particularly problematic in that individual differences in cognitive processing that affect these three 4 "routes" to creative thought probably are not independent. For example, the looseness in perceptual organization that may characterize creative individuals with schizotypal thought patterns may well be negatively correlated with ability to make effortful mental transformations as well as the ability to recognize that a novel mental representation is worthwhile. We do believe, however, that research efforts into individual differences in specific perceptual characteristics potentially can be useful in both increasing our understanding of their relationships to creative behavior, as well as for determining circumstances that might foster creative thought. As we have noted, research literature describing individual differences in perceptual organization tendencies is notably lacking. The study of individual differences in executively controlled transformations of mental representations has received considerably more research effort, due to a long existing presumption that such operations are closely related to measures of basic mental abilities. However, the "mental measurement" motivation for much of this research, has, in our view, directed researchers more toward the study of "common transformational ability factors" (e.g., Guilford, 1981, 1983) rather than toward the properties of specific types of mental code manipulations, such as synesthetic metaphor. Lastly, the literature on spontaneous generation of novel representations by perceptual mechanisms is, at present, highly speculative, and consists primarily of self-report anecdotes.

\section{A Flow Diagram of the Creative Process and Its Implications}

To summarize our view of how processes related to perception influence the creative process, we offer the flow diagram shown in Figure 6. In addition, Table 1 summarizes some of our conjectures about the relationships between various components of the flow diagram and some individual difference variables potentially measurable by existing or designable assessment instruments and surveys.

Essentially, Figure 6 is a visual summary of our previous discussions of a configural view of the creative process. It lists as sources of novel representations (1) atypical involuntary processes of perception, including both loose organizational processes 


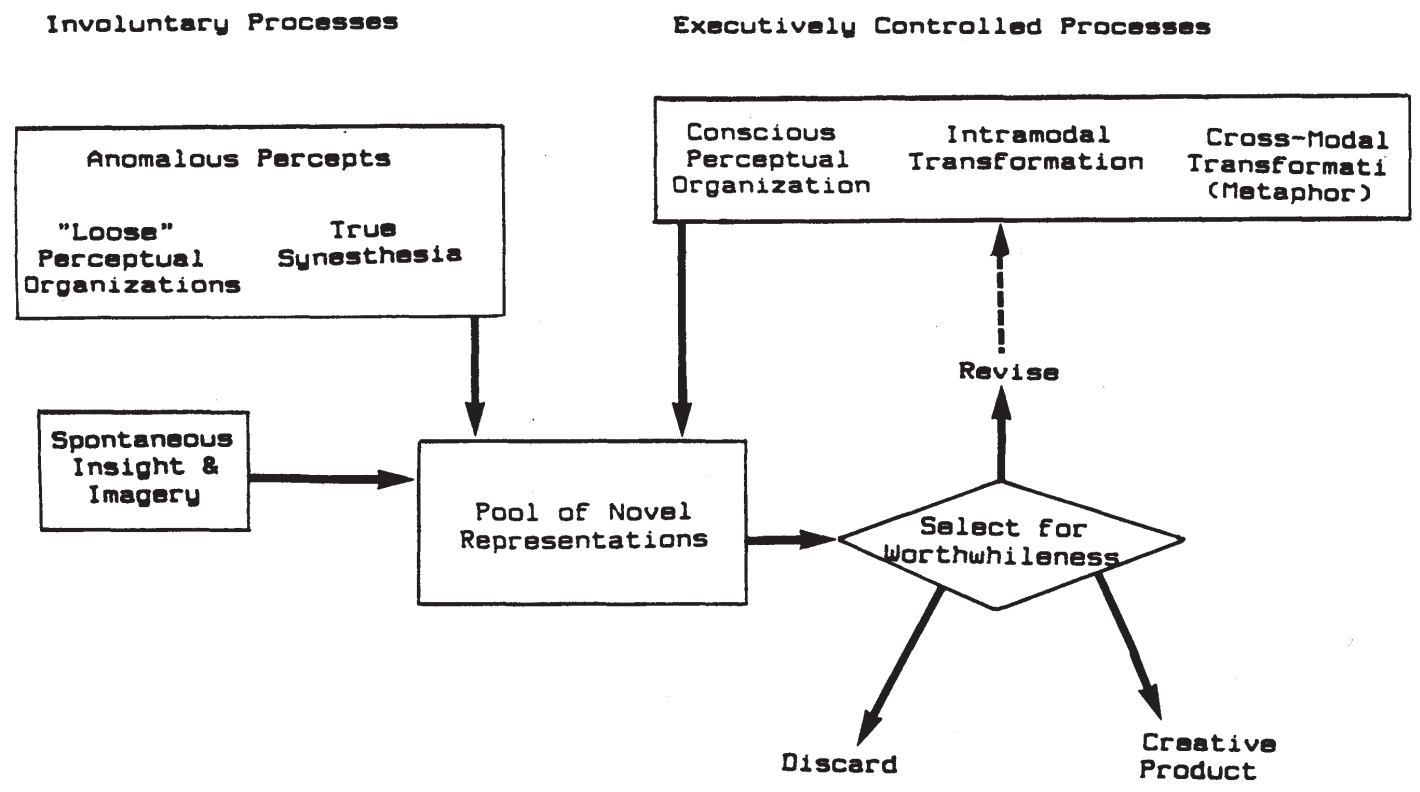

Figure 6. A schematic representation of our view of how involuntary and executively controlled perceptual processes contribute to creative thought (see text for details).

and true synesthesia, (2) spontaneous (and involuntary) generation of insight and imagery, (3) conscious perceptual organization, (4) conscious intermodal transformations of representations, and (5) conscious cross-modal transformations of representations. In addition, it includes the process of selection of novel representation and feedback to the executively controlled processes for purposes of refining the potential creative product.

In Table 1, we have chosen to relate the uses of each of the potential sources of novel representations discussed in this chapter together with an "efficiency" " selection mechanism drawn from the following set of individual differences variables: (1) strength of per- ceptual organization, SPO; (2) imagery ability, IM; (3) general measures of transformation ability, T; (4) schizotypal thought, ST; (5) drug usage, DU; (6) ability to report dreams, DR; and (6) amount of reported conscious effort expended on the problem for which a creative solution is found, EF. Note that these items constitute a nonexhaustive sample of potentially measurable individual differences variables. As we have mentioned previously, respected psychometric instruments exist that presumably tap some of these, whereas for others, instruments would need to be developed. Furthermore, measurement of some of the self-report variables (such as DR and EF) would most likely involve both validity and reliability problems

Table 1. Hypothetical Associations between Modes of Generating and Selecting Creative Representations and Various Measures of Individual Differences (see text for a detailed explanation).

\begin{tabular}{llllllll}
\hline Source & SPO & IM & T & ST & DU & DR & EF \\
\hline Loose perceptual organization & - & - & - & + & $+?$ & $?$ & $-?$ \\
Synesthesia & $?$ & $+?$ & $?$ & $-?$ & + & $?$ & $?$ \\
Spontaneous imagery & $+?$ & + & + & $-?$ & $?$ & + & + \\
Conscious perceptual organization & + & + & + & - & $-?$ & $?$ & + \\
Intramodal transformation & + & + & + & - & - & $?$ & + \\
Cross-modal transformation & $?$ & $+?$ & $?$ & - & $?$ & $?$ & $+?$ \\
\hline
\end{tabular}


that we will not address here. Given those disclaimers, and with the additional disclaimer that nearly all the cell entries are based upon our own current personal speculations as opposed to existing data, we offer this table of hypotheses as a guide for potential future research. The cell entries themselves consist of + and - signs to indicate presumed positive and negative correlations, together with ? signs that specify either that no particular relationship is likely to exist, or, if there is one, we have no basis to speculate its directionality. Additionally, we have used the question mark as a modifier to indicate above average uncertainty about our speculations.

We should call attention to one pattern among these hypothetical intercorrelations that emphasizes our previous statements about the configural nature of creativity. That pattern is that individuals whose creativity stems largely from anomalous perceptual inputs (i.e., individuals with loose perceptual organization and perhaps a rare, creative, true synesthetic) are likely to be very different from creative individuals who rely more heavily upon executively controlled processes. Conceivably, this might bear upon differences in the types of creative products that are developed by individuals who rely predominantly upon anomalous percepts for novel representations, as opposed to those arising from some combination of executively controlled manipulations of representations and spontaneous imagery and/or insight. As a possible case in point, one often encounters examples of schizophrenic paintings and drawings, yet rarely does one encounter mention of schizophrenic poetry or sculpture.

As a final point, we do not wish to imply, by our apparent emphasis upon individual difference variables in this final section of this chapter, that research on conditions that affect the likelihood of creative behavior within a given individual is less important than research aimed at predicting patterns of creativity among individuals. Clearly, the two classes of research complement each other; by learning more about relationships between perception-related processes as varieties of creative behavior, we may discover strategies for optimizing creative production within individuals. Similarly, through applied research aimed at development of "thinking aids," "designing aids," and "writing aids," or through the evaluation of such products, we may discover additional relationships among perceptual and cognitive processes and the creative process.

\section{References}

Brooks, L. R. (1968). Spatial and verbal components of the act of recall. Canadian Journal of Psychology, 22, 349-368.

Gibson, J. (1966). The senses considered as perceptual systems. Boston: Houghton Mifflin.

Goodman, K. J., \& Marquart, D. I. (1978). Creativity and perception: The neglected theory of Lowenfeld. Journal of Creative Behavior, 12, 279.

Guilford, J. P. (1967). The nature of human intelligence. New York: McGraw-Hill.

Guilford, J. P. (1981). Higher order structure of intellect abilities. Multivariate Behavioral Research, 16, 411-435.

Guilford, J. P. (1983). Transformation abilities or functions. Journal of Creative Behavior, 17, 75-83.

Hochberg, J. (1981). Levels of perceptual organization. In M. Kubovy \& J. Pomerantz (eds.), Perceptual organization (pp. 255-278). Hillsdale, NJ: Erlbaum.

Kahneman, D., \& Tversky, A. (1973). On the psychology of prediction. Psychological Review, 80, 237-251.

Keefe, J. A., \& Magaro, P. A. (1980). Creativity and schizophrenia: An equivalence of cognitive processing. Journal of Abnormal Psychology, 89, 390-398.

Kellogg, R. (1986). Designing ideal processors for document composition. Behavior Research Methods, Instruments, and Computers, 18, 118-128.

Kohler, W. (1947). Gestalt psychology. New York: Liveright.

Marks, L. (1978). The unity of the senses. New York: Academic Press.

Marks, L. (1982). Synesthetic perception and poetic metaphor. Journal of Experimental Psychology: Human Perception and Performance, 8, 15-23.

Newman, S. S. (1933). Further experiments in phonetic symbolism. American Journal of Psychology, 45, 53-75.

Paivio, A. (1971). Imagery and verbal processes. New York: Holt, Rinehart \& Winston.

Paivio, A. (1978). Comparison of mental clocks. Journal of Experimental Psychology: Human Perception and Performance, 4, 61-71.

Place, E., \& Gilmore, G. (1980). Perceptual organization in schizophrenia. Journal of Abnormal Psychology, 89, 409-418.

Pomerantz, J., \& Schwaitzberg, S. (1975). Grouping by proximity: Selective attention measures. Perception and Psychophysics, 18, 355-361.

Pomerantz, J., Sager, L., \& Stover, R. (1977). Perception of wholes and their component parts: Some configural superiority effects. Journal of Experimental Psychology: Human Perception and Performance, 3, 422-435. 
Prentky, R. A. (1979). Creativity and psychopathology: A neurocognitive perspective. In B. Maher (ed.), Progress in experimental personality research (Vol. 9, pp. 1-39). Academic Press.

Sapir, E. (1929). A study of phonetic symbolism. Journal of Experimental Psychology, 12, 225-239.

Segall, S. J., \& Fusella, V. (1970). Influence of imaged pictures and sounds in detection of visual and auditory signals. Journal of Experimental Psychology, 83, 458-474.

Shepard, R. N. (1978). Externalization of mental images and the act of creating. In B. Randhawa \& W. Coffman (eds.), Visual learning, thinking, and communication (pp. 133-189). New York: Academic Press.

Shepard, R. N. (1981). Psychophysical complementarity. In M. Kubovy \& J. Pomerantz (Eds.), Perceptual organization (pp. 279-341). Hillsdale, NJ: Erlbaum.
Stein, M. I. (1956). A transactional approach to creativity. In D. W. Taylor (ed.), Research conference on the identification of creative talent. Salt Lake City: University of Utah Press.

Stevens, S. (1959). Cross-modality validation of subjective scales for loudness, vibration and electric shock. Journal of Experimental Psychology, 57, 201-209.

Taylor, D. W. (1960). Thinking and creativity. Annals of the New York Academy of Sciences, 91, 108-127.

Wells, D., \& Leventhal, D. (1984). Perceptual grouping in schizophrenia: Replication of Place \& Gilmore. Journal of Abnormal Psychology, 93, 231-234. 